\title{
Ethosomal formulation composed of standardized Orthosiphon stamineus extract and sophorolipid inhibits angiogenesis of melanoma in vivo
}

\author{
Mansoureh Nazari Vishkaei ${ }^{a}$, Mohamed Khadeer Ahamed Basheer ${ }^{c}$, Amin Malik Shah Abdul Majid ${ }^{a, b}$
}

\begin{abstract}
Cancerdisease involves the abnormal growth of cells that have the ability to invade other organs. Melanoma is one of the angiogenesis dependent cancer. Orthosiphon stamineus (EXOS) extract is a well-known antiangiogenic herb in South Asia. Thin-film hydration method has been employed to develop ethosome, a flexible liposomal gel formulation composed of standard extract of O.S leaves (SLOS) using sophorolipid (SL) and investigated its physicochemical properties for topical drug delivery system. High Performance Liquid Chromatography (HPLC) determined 76.02.02 $\pm 1.2 \%$ entrapment of rosmarinic acid (RA) in ethosome vesicle. The average particle size of optimized vesicle was 189.9 $\mathrm{nm}$ with potential of $-35.5 \mathrm{mV}$. SLOS at concentration of $1 \%$ demonstrated proper plastic flow, which could facilitate achieving effective and maximum skin permeation of EXOS. Permeation studies using albino mice revealed that the SLOS demonstrated higher penetrability through deeper layers of skin than the conventional hydroethanolic gel composed of EXOS. Cytotoxic test on normal endothelial cells confirmed the safety of SLOS formulation. In vivo melanoma tumor model study revealed that SLOS showed $97.07 \pm 0.6 \%$ anti
\end{abstract}

Significance | SLOS gel is a novel topical formulation and a promising treatment for skin cancer.

\section{*Correspondence: Amin Malik Shah Abdul Majid at Department of Pharmacology, School of Pharmaceutical Sciences, Universiti Sains Malaysia, Minden, 11800 , Pulau Penang, Malaysia, Tel: +60124230842 Email: aminmalikshah@gmail.com}

Edited by Md. Asaduzzaman Shishir, University of Dhaka. And accepted by the Editorial Board February 4, 2019 (received for review January 17, 2019) -tumor activity during 16 days of study. These results revealed that SLOS vesicles can be considered for effective dermal therapeutic agent for the treatment of skin cancer.

Keywords: Ethosome; sophorolipid; Orthosiphon stamineus, Melanoma; Rheology; Topical drug delivery

\section{Introduction}

Melanoma is one the most aggressive types of skin cancer. Malignant melanoma has been reported as an angiogenic tumor type, unmistakably showing new vessel arrangement as an essential advance in infection movement from atypical melanocytes, through outspread development to the forceful vertical development stage (Vishkaei, Sultan, Basheer, \& Majid, 2017)(Vishkaei, Sultan, Basheer, \& Majid, 2017). Orthosiphon stamineus Benth is well known therapeutic herb in south Asia since long time ago for the treatment of bladder inflammation, eruptive fever, hepatitis, hypertension, and rheumatism (Hossain \& Rahman, 2015; Vishkaei, Sultan, Khadeer, \& Ahamed, 2017). It is abundant with flavonoids with recognized anti-oxidant, anti-inflammatory anti-tumor and anti-angiogenic properties (Shafaei et al., 2016). For the treatment of skin diseases, topical drug delivery is one of the desired types of administrations. Due to the selective permeable characteristics of outer layer of skin, which acts as

\section{Author Affiliation:}

a Department of Pharmacology, School of Pharmaceutical Sciences, Universiti Sains Malaysia, Minden, 11800 Pulau Penang, Malaysia

${ }^{b}$ ACRF Department of Cancer Biology and Therapeutics, the John Curtin School of Medical Research, Australian National University.

${ }^{C}$ EMAN Biodiscoveries Sdn. Bhd.,Suite 126, Level 1, EUREKA Complex, Universiti Sains Malaysia (USM) Campus, Minden - 11800, Pulau Penang, Malaysia

\section{Please cite this article:}

Mansoureh Nazari Vishkaei, Mohamed Khadeer Ahamed Basheer, Amin Malik Shah Abdul Majid. (2019). Ethosomal formulation composed of standardized Orthosiphon stamineus extract and sophorolipid inhibits angiogenesis of melanoma in vivo. Angiotherapy, 1(2), pages 087-096.

2207-8843/๑ 2019 ANGIOTHERAPY, a publication of Eman Research Ltd, Australia. This is an open access article under the CC BY-NC-ND license. (http://angiotherapy.emanresearch.org) 
a stringent barrier, in many conditions, permeation enhancers are required to maintain therapeutic quantities of medicine. Traditional liposomal techniques were identified to be efficient at topical drug delivery for the purpose of skin therapy (Bragagni, Scozzafava, Mastrolorenzo, Supuran, \& Mura, 2015). However, the conventional liposomal technique has several major drawbacks, such as low drug entrapment, low drug-holding capability, low stability and inefficient drug delivery to the deeper level of the skin In contrast, ethosomal particles through the phospholipid vesicular techniques that include high percentage of alcohol, were efficient at improving dermal and transdermal delivery of either lipophilic or hydrophilic compounds (Mbah, Builders, Agubata, \& Attama, 2017). Ethosome vesicles are phospholipid-based elastic nano particles including high concentrations of ethanol (20-45\%). Ethanol is identified as an effective permeation booster and has been integrated in the vesicles to make the elastic nano particles. It can mingle with the polar head group part of the lipid substances, causing the elimination of the melting point of lipid in the structure of the stratum corneum, consequently raising lipid fluidity, as well as permeability of cell membrane. The superior flexibility of vesicular membranes is attributed to the presence of ethanol in ethosome. This enables the vesicles to become more elastic and therefore can be easily absorbed into the skin layers by squeezing themselves through the pores of even smaller dimensions. Thereby, in transdermal drug delivery system with respect to the effective amount and depth of penetration, ethosomal technology is significantly more effective than either traditional liposomes or hydroalcoholic formulations (Malla et al., 2016). Some studies have also specified that ethosomal systems also own perfect stability of entrapment due to the fact that the existence of ethanol, that produces a negative surface charge, consequently hindering vesicle aggregation because of electrostatic repulsion. Ethosomal drug delivery system has proved safe and effective in human study, wherein 5-fluorouracil has shown promising result in penetration through human skin (Vishkaei, Sultan, Basheer, et al., 2017)

The main aim of this research work was to develop an optimized topical ethosomal-based formulation of O.S extract with enhanced rate of penetration through deeper layers of skin and treat melanoma tumor model via topical application.

\section{Materials and methods}

Sophorolipid was given as a gift from Malaysian palm oil board. Orthosiphon stamineus standard extract was given as gift from natureceuticals.sdn.bhd.Pulau penang, Malaysia. Solvents of either HPLC or analytical grade were purchased from Merck. Rosmarinic Acid and other markers and rhodamine red from Sigma-Aldrich, USA,DMEM media Gibco/Life technology, UK and B16F10 cell line from ATCC, Rockville, MD, USA.

\section{Animals}

For permeation and tumor model studies healthy adult male Albino mice weighing 28-32 g were used. The study was approved by the
Animal Ethics Committee of Universiti Sains Malaysia, Penang, Malaysia [Protocol No: USM/IACUC/2017/ (109) (882)].

\section{Preparation of ethosome dispersion}

Ethosome formulation (SLOS) developed using thin film rehydration method with some modifications. Different ratio of phospholipids: O.S extract (EXOS) (2:1, 1:1 and 1:2) were suspended in $15 \mathrm{~mL}$ of $50 \%$ ethanol. The mixture was homogenised for $10 \mathrm{~min}$ at high-speed shearer/mixer. This mixture was agitated at $120 \mathrm{rpm}$ in a shaking incubator for 3 hours at $40{ }^{\circ} \mathrm{C}$. The final solution was concentrated using a rotary evaporator to prepare a thin film. Then, $40 \%$ ethanol added and covered it properly to avoid evaporation of ethanol and kept it in incubator shaker with $120 \mathrm{rpm}$ for 1 hour. It was analysed for the entrapment percentage of different formulation using cold centrifuge method to select the best formulation for further studies (Rezaei-Sadabady, Eidi, Zarghami, \& Barzegar, 2016).

\section{Preparation of ethosome gel formulation:}

The selected dispersion was chosen to make $1 \%$ and $2 \%$ gel utilizing acrypol. 1 and $2 \% \mathrm{~W} / \mathrm{V}$ acrypol added gradually to the beaker contained selected dispersion of ethosome while stirring continuously. The $\mathrm{pH}$ of dispersion controlled to be in neutral $\mathrm{pH}$ using sodium hydroxide. After 1hour collected the gel and kept in fridge (Ramadon, Goldie, \& Anwar, 2017).

\section{Characterization}

\section{Entrapment percentage}

Entrapment efficiency of ethosomal formulation was assessed by defining the content of rosmarinic acid entrapped in the ethosomal vesicles using UV spectrophotometer at wavelength of $320 \mathrm{~nm}$ (Iizhar, Syed, Satar, \& Ansari, 2016).

\section{Particle sizes and zeta potential}

The average particle size, PDI and zeta potential of selected formulation was assessed by a Zetasizer Nano ZS (Malvern Instruments Ltd, UK).

\section{HPLC}

HPLC analysis performed on RA as standard compound and SLOS using previously validated method 9. A RP-HPLC-diode-array detector at a $320 \mathrm{~nm}$ wavelength used to detect the marker compound (RA) and to ensure accuracy, precision, and sensitive detection. The assay conducted to quantify RA in SLOS compared with EXOS prepared in ethanol, using a gradient mobile phase of $0.1 \%$ formic acid in Acetonitrile at a flow rate of $1 \mathrm{~mL} / \mathrm{min}$ in a reverse-phase acclaim polar advantage II C18 column $(3 \mu \mathrm{m}, 3 \times 150 \mathrm{~mm})$ with the separation time being $20 \mathrm{~min}$ (Shafaei, Ab Halim, Zakaria, \& Ismail, 2017).

\section{Transmission electron microscopy (TEM)}

TEM study was done in order to verify the formation of SLOS. The samples were studied under CM12 TEM (Philips, Netherlands).

\section{Zeta sizer and potential}

The average particle size, PDI and zeta potential were determined by a Zetasizer Nano ZS (Malvern Instruments Ltd, UK). 
Viscosity analysis of ethosome gel in different temperatures

MCR 302 Rheometer (Anton Paar, Malaysia) along with Parellel Plate PP25 (Diameter 25mm, Stainless steel) was used to study rheological analysis of SLOS. The temperature was controlled by Plate Peltier Temperature Controller P-PTD200. Software Anton Paar Rheocompass version 1.2. Prior to the rheological experiment SLOS gel was placed on the peltier plate in the way to cover the cone at the top of the plate. Then the SLOS gel was equilibrated at a controlled temperature dependent on the experimental condition for 3 minutes before and after putting the cone over Peltier plate. After fixing the cone trimmed the gap by using this option in rheometer to obtain thin uniform film of SLOS gel under the cone. After that without disturbing the film between plate and cone removed the excess of gel from sides of the cone carefully.

\section{Continuous flow ramp study}

SLOS gels $1 \%$ and $2 \%$ were kept in the condition to increase shear rate from 1-200 S-1 and the corresponding stress was analyzed at $4{ }^{\circ} \mathrm{C}, 25$ ${ }^{\circ} \mathrm{C}$ and $32^{\circ} \mathrm{C}$.

\section{Drug content}

$10 \mathrm{ml}$ of SLOS gel was dissolved in $1 \mathrm{ml}$ methanol and stirred for 30 min. Drug content was quantified using HPLC (Li et al., 2018).

\section{Cell Viability study}

Cytotoxicity of EXOS and SLOS were evaluated against endothelial cell line (EA.hy926) using 3-(4,5-dimethylthiazol-2-yl)- 2,5diphenyltetrazolim bromide (MTT) assay as described earlier. The experiment was repeated three times with four replicates for each concentration. The percentage of cell viability was calculated and presented as mean \pm S.E.M.

\section{Ex vivo rat aortic ring assay}

The rat aortic ring assay was carried out with slight modification $(\mathrm{Ng}$, Salhimi, Majid, \& Chan, 2010). Briefly, aortic rings (1 mm thickness) taken from thoracic aortas of 12-14 weeks old male Sprague Dawley rats were seeded individually in 48 -wells plate in $300 \mu \mathrm{l}$ serum free M199 media containing $3 \mathrm{mg} \mathrm{ml-1}$ fibrinogen and $5 \mathrm{mg} \mathrm{ml}-1$ aprotinin. $50 \mathrm{NIH} \mathrm{U} \mathrm{ml-1} \mathrm{thrombin} \mathrm{in} 0.15 \mathrm{M} \mathrm{NaCl}$ were added in each well. After $90 \mathrm{~min}$ incubation at $37^{\circ} \mathrm{C}$, equivalent concentrations of SLOS and EXOS dissolved in $0.3 \mathrm{ml} \mathrm{M} 199$ medium supplemented with $20 \%$ HIFBS, $0.1 \% \dot{\varepsilon}$-aminocaproic acid, $1 \%$ L-Glutamine, 2.5 $\mu \mathrm{g} / \mathrm{ml}$ amphotericin $\mathrm{B}$, and $60 \mu \mathrm{g} / \mathrm{ml}$ gentamicin, were added to each well. Suramin and $1 \%$ ethanol were used as positive and negative controls, respectively. On day four, the medium was replaced with a fresh one containing the compound. On day five, aortic rings were photographed at $4 \mathrm{x}$ magnification using an inverted light microscope (Olympus). The angiogenic response was determined by measuring the distance of blood vessels outgrowth from the primary tissue explants using the same instrument with the aid of Leica Quin software package. The results are presented as mean percent inhibition to the negative control \pm S.E.M, $(\mathrm{n}=3)$.

\section{Ex vivo skin Permeation study}

In this study, a Franz diffusion apparatus was used with minor modifications to obtain an efficient diffusion surface area of about 2 https://doi.org/10.25163/ angiotherapy.1120591141830219 $\mathrm{cm}^{2}$. In this experiment, $2 \times 2 \mathrm{~cm}$ area of mouse abdominal skin was shaved until all fur was removed. Then the cleaned and shaved skin explants were excised from abdominal regions of albino mice. The skin explant was mounted as a barrier between the donor compartment and the receptor compartment of the Franz diffusion apparatus. Test sample (SLOS or EXOS) was applied on the mounted skin surface in the donor compartment and covered with parafilm to avoid evaporation of ethanol. The receptor section of the diffusion cell was filled with phosphate buffer $\mathrm{pH}$ 7.4. The entire setup was installed on a magnetic stirrer, so that the solution in the receptor section was continuously stirred by means of magnetic beads at $50 \mathrm{rpm}$. The temperature was maintained at $32 \pm 0.5^{\circ} \mathrm{C}$ to mimic skin body temperature. At specific time intervals for $24 \mathrm{~h}$, samples were taken and the quantity of rosmarinic acid content was estimated spectrophotometrically at $320 \mathrm{~nm}$, as explained earlier. In the receptor section an equivalent quantity of fresh phosphate buffer $\mathrm{pH} 7$ was replaced at each time interval. The analysis was done in triplicate using different skin explants from 3 animals for each test sample for each time point $(n=3)$. Average percentage \pm S.E.M of drug permeated through the skin over time was plotted.

In vivo melanoma tumor model in albino mice model

B16Fl0 melanoma cells were injected subcutaneously into the right flank of each mouse (4-6 week old, 20-22g) C57BL/6 mice.

The animals were divided to 3 groups of 6 animals each. Group one was treated with empty ethosome topical gel as negative control. Group two and three were applied $1000 \mathrm{mg} / \mathrm{kg}$ of SLOS and EXOS respectively at equivalent concentration once per day. Various parameters i.e., tumor size and body weight were identified every 3 days and tumor volume was measured using the following formula; Tumor volume $(\mathrm{mm})^{3}=(\mathrm{L} \times \mathrm{W} \times \mathrm{D}) / 2$

While L, W and D stand for length, width and depth of tumor in $\mathrm{mm}$ respectively.

The anti-melanoma effect of ethosomal formulation of O. stamineus was analyzed using following equation;

\section{Anti-melanoma efficacy $=\% \Delta \mathrm{T} / \Delta \mathrm{V}$}

$\Delta \mathrm{T}=\mathrm{T}-\Delta 0$ and $\Delta \mathrm{C}=\mathrm{C}-\Delta 0$ ( $\mathrm{T}, \Delta 0$ and $\mathrm{C}$ is equal to average of tumor volume at $\mathrm{t} 0$, in different days and in various treated and untreated groups.

All the animals were euthanized after 4 weeks when the tumor in untreated group exceeded to more than $1000 \mathrm{~mm}^{3}$. Eventually the survived animals were euthanized using $\mathrm{CO}_{2}$, then cervical dislocation was done. The images of animals were taken by camera Canon and the tumor were collected and preserved in $4 \%$ paraformaldehyde. The tumors were sent to Gribbles pathology laboratories (Penang, Malaysia) to do haematoxylin/EXOS in staining in order to observe them under microscope to visualize the apoptosis/necrosis caused by the treatment after finishing the period of the study.

\section{Statistical analysis}

Data are expressed as the mean \pm S.E.M. Statistical analysis was performed using ANOVA test with graph prizm software. The level of probability at 0.05 was used as the level of significance. 


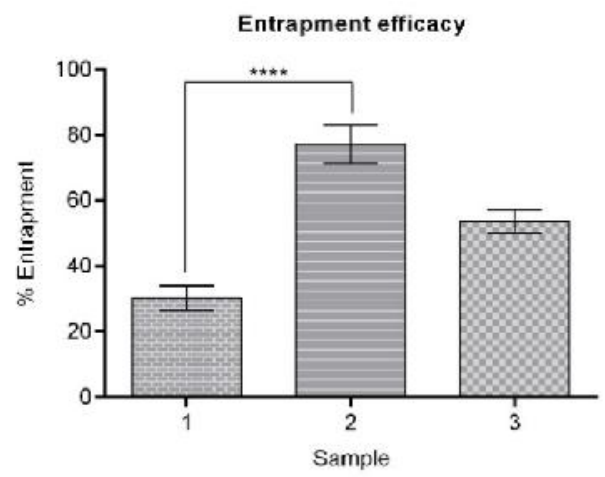

Figure 1 | Entrapment percentage of SLOS.

Ethosomes were formulated by mixing $0 . S$

ethanol-water extract with sophorolipid

with different ratios (1-3).

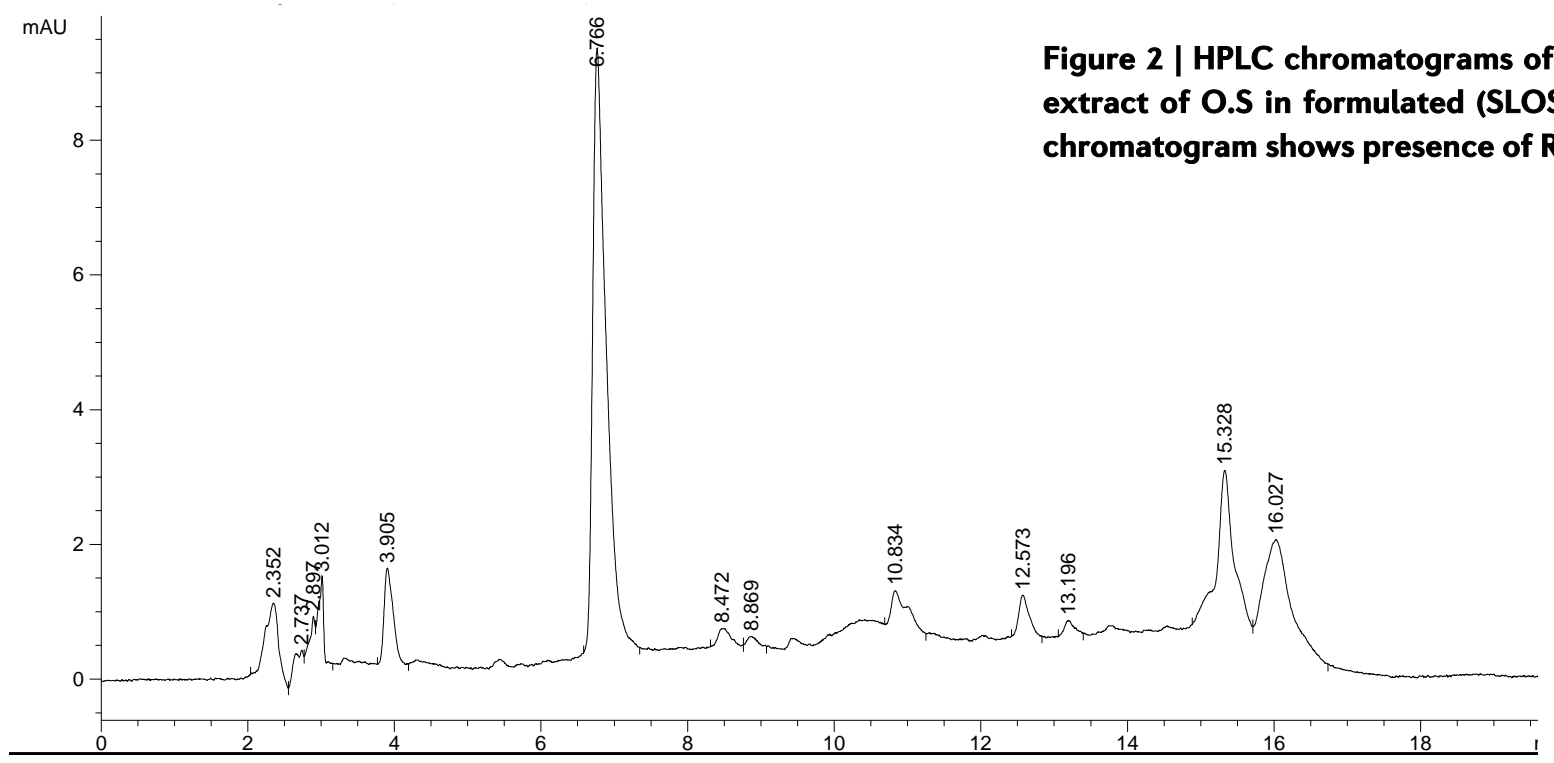

\section{Results and discussion}

Entrapment analysis

UV spectroscopy analysis revealed that the entrapment efficiency was less in formulation-1 composed of ratio 1:2 of sophorolipid: extract was $30.38 \pm 3.78, \mathrm{n}=3$. After that formulation-2 which is composed of ratio 2:1 of extract: sophorolipid. Figure 1 shows the percentage of RA entrapped in formulations. However, the formulation-2 which is composed of sophorolipid: extract at a 1:1 ratio, showed significantly $(P<0.0001)$ highest entrapment efficiency with 77.23 \pm 5.9 . Based on the entrapment results, formulation- 2 was chosen for the further physico-chemical and pharmacological characteristic studies.

HPLC standardization of O.S-ethosome formulation and crude extract

HPLC analysis of rosmarinic acid (RA) (Figure2) enabled the determination of the extent of active compound entrapment in the SLOS compared with EXOS. These results confirmed entrapment analysis using UV. It showed $76.02 \pm 1.2 \%$ RA entrapped in ethosomal selected formulation.

\section{TEM}

The SLOS formulation was dissolved in water and studied under TEM. Figure 3 shows the presence of the predominantly roundshaped particles of the SLOS. However, few flat depressions on the surface of most of the particles suggest the flexible nature of the ethosomal particles.

https://doi.org/10.25163/ angiotherapy.1120591141830219

\section{Zeta sizer and potential}

Zeta sizer has shown SLOS with the diameter of $189.9 \mathrm{~nm}$ with PDI of 0.404 (Figure $4 \mathrm{~B}$ ). The zeta potential showed $-35.5 \mathrm{mv}$ (Figure $4 \mathrm{~A}$ ). Rheological study

\section{Continuous flow ramp study}

The 1\% SLOS gel showed a suitable plastic flow behavior with yield stress of approximately $60.099 \pm 4.20 \mathrm{~Pa}$ at low concentration of polymer at $4{ }^{\circ} \mathrm{C}$. Figure 5 shows the changes in yield stress by increasing the temperature as well as changing the concentration of acrypol. Viscosity of samples was decreased by increasing the temperature while increasing the shear rate. Comparatively, viscosity of $2 \%$ gel in all the conditions was recorded to be more than the $1 \%$ SLOS gel.

The SLOS gel, irrespective of acrypol concentration demonstrated good spreadibility and was free of agglomerates. The rheological properties of SLOS gels was analysed to select proper acrypol percentage and application. For dermal and transdermal formulations, the proper SLOS gel must have plastic flow (Jain, Patel, Madan, \& Lin, 2016). Results of viscosity analysis demonstrated that $1 \%$ SLOS gel proved to be more pronounced than the formulation composed of $2 \%$ gel, with respect to the transdermal penetration.

\section{Effect of SLOS on Cell viability}

Cytotoxicity effect of SLOS formulation on normal cell line (EA.hy926) showed that SLOS did not affect the viability of the cells. The test was conducted utilizing colorimetric measurement of cell E087-E096 | ANGIOTHERAPY | Published online February 4, 2019 


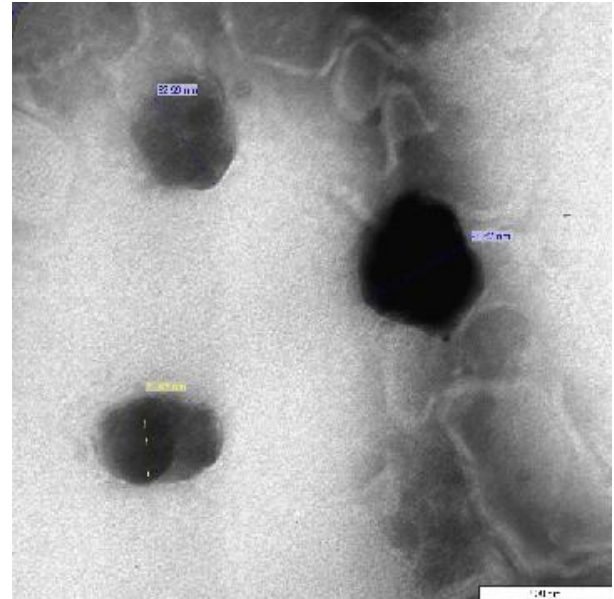

Figure 3 | The presence of the predominantly round-shaped particles of the SLOS.
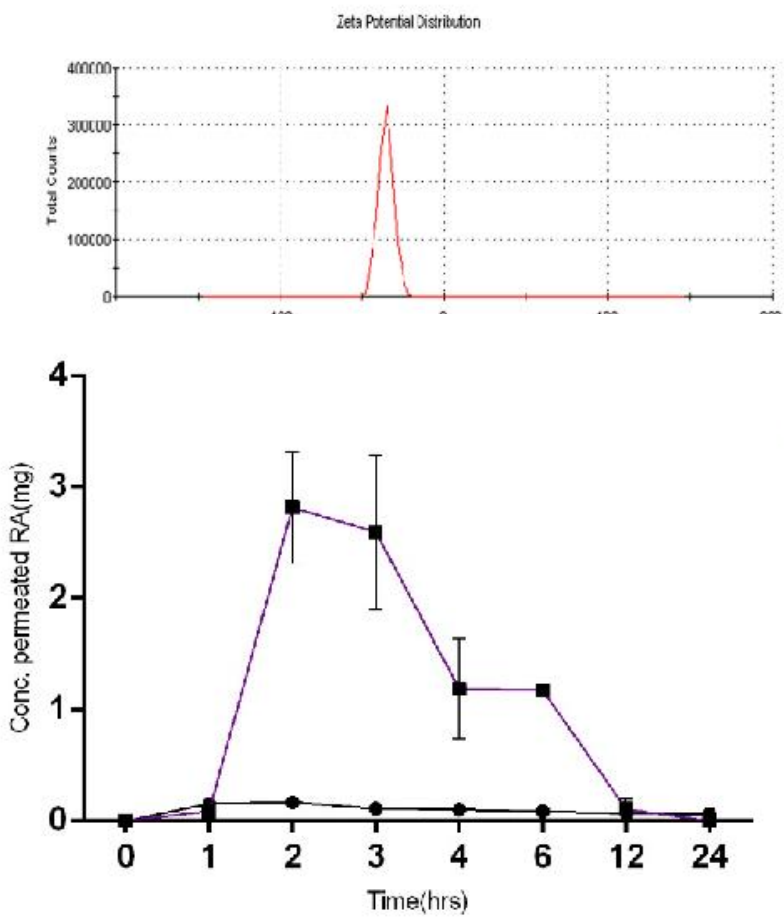

viability after 48 hours treatment with SLOS compared to EXOS crude extract at concentrations 100 and $50 \mu \mathrm{g} / \mathrm{mL}$. Figure 6 demonstrates the percentage of EA.hy 926 cell viability after treatments.

\section{Ex vivo Permeation study}

In this study, $1 \mathrm{~g}$ of the test product was applied onto the isolated skin mounted on Franz diffusion cell. After 24 hours, the transdiffused solution was collected and the marker compound of EXOS was quantified using HPLC. The results of the test showed that the concentration of RA found in the transdiffused solution after application of the hydroethanolic gel of EXOS and the SLOS gel was $0.11 \pm 0.009 \mu \mathrm{g} / \mathrm{ml}$ and $0.23 \pm 0.01 \mu \mathrm{g} / \mathrm{ml}$, Figure : Permeation studies. Permeation of EXOS and SLOS gel in 24 hours of topical application mice skin. The data is presented as mean $\pm S E M, n=3$.

respectively (Figure 4.B). This result indicates the SLOS has shown 2 times more permeability than that of EXOS. So based on enhance permeation efficacy of formulation it has the potential to be used for topical application in vivo study.

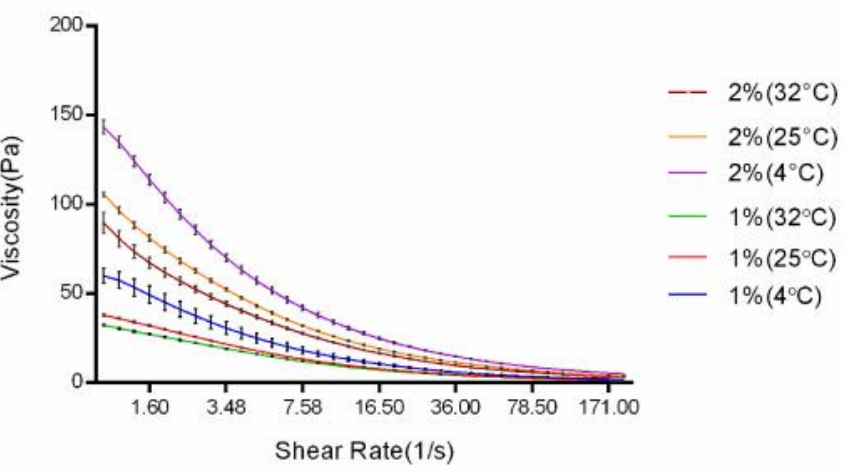

Figure 5 | The effect of shear rate on viscosity of $1 \%$ and $2 \%$ gel of SLOS in $4^{\circ} \mathrm{C}, 25^{\circ} \mathrm{C}$ and $32{ }^{\circ} \mathrm{C}(\mathrm{n}=3)$.

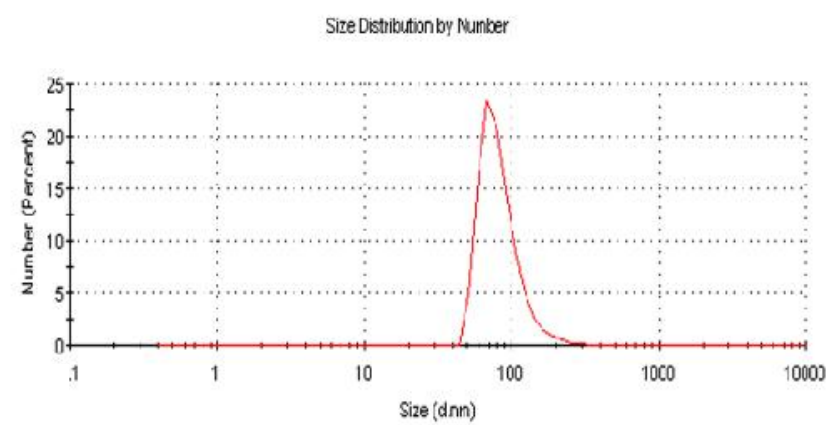

Figure 4 | (A) Zeta potential (B) Zeta sizer results of SLOS sample after dissolving in water.

\section{Ex vivo rat aortic ring assay}

The anti-angiogenic potential of SLOS compared with EXOS was investigated in the rat aortic model. Figure 7 shows the microvessels outgrowth from the untreated aortic rings. On the contrary, aortic rings treated with SLOS exhibited sopped outgrowth at concentration of $100 \mu \mathrm{g} / \mathrm{ml}$ (Figure 7B). The anti-angiogenic effect on explants of rat aorta showed a significant dose dependent relationship $(\mathrm{P}<0.05)$. At $50 \mu \mathrm{g} / \mathrm{ml}$, SLOS and EXOS inhibited vascularisation by $58 \pm 5.08$ and $34.61 \pm 1.8 \%$ respectively. Doubling the dose of SLOS and EXOS to 100 $\mu \mathrm{g} / \mathrm{ml}$ led to a complete inhibition of angiogenesis by $100 \%$ for SLOS but $74.44 \pm 5.20 \%$ inhibition by EXOS. Suramin, which was used as a positive control showed $100 \%$ inhibition of microvessels outgrowth at $100 \mu \mathrm{g} / \mathrm{ml}$ (Figure 7F).

In this study, angiogenesis assays was employed to evaluate the antiangiogenic activity of SLOS compared with EXOS. The results showed that SLOS perturbed the new blood vessels formation in rat aortic ring explants $25.56 \%$ more than EXOS at high concentration of $100 \mu \mathrm{g} / \mathrm{ml}$ (Figure 7). 

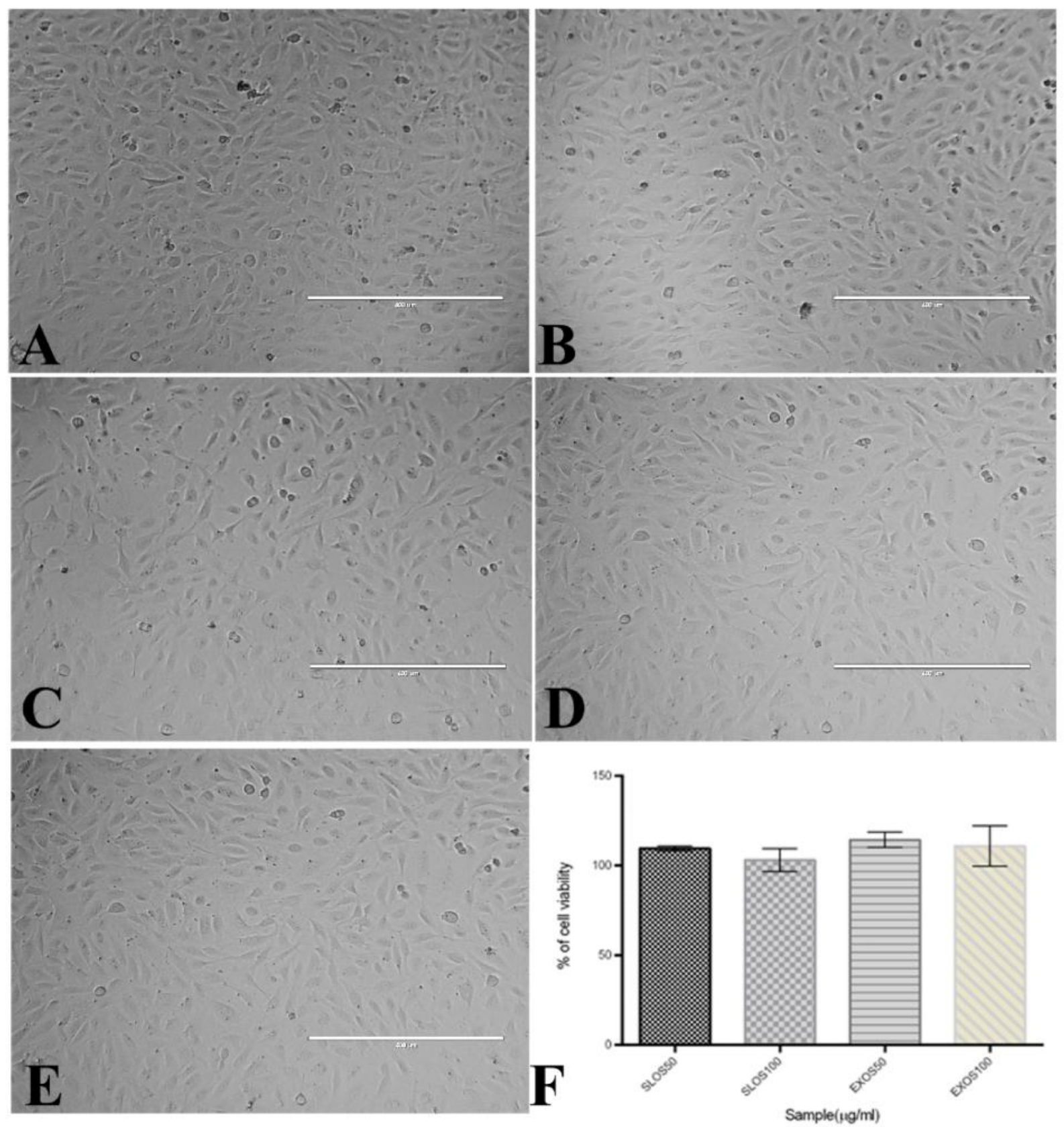

Figure 6 | (A, B) EA.hy926 cell line treated with SLOS at concentration 50 and $100 \mu \mathrm{g} / \mathrm{mL}$.(C,D) treated with EXOS equivalent concetration of 50 and $100 \mu \mathrm{g} / \mathrm{mL}$. (E) negative control. Percentage of EA.hy926 cell line viability after treatment. SLOS and EXOS refer to sophorolipid ethosomal formulation and crude extract of O.S, respectively. Statistical analysis was conducted by one-way ANOVA.

\section{In vivo anti-melanoma tumor model}

The in vivo antitumor effect of topical SLOS evaluated compared with EXOS by inducing the B16F10 cells subcutaneously in albino mice. After 6 days the treatment based on dosage forms of SLOS and EXOS were started (Figure 8). Figure 8 demonstrated enhanced antitumor effect of SLOS compared with EXOS gel in 16 days of treatment $(\mathrm{P}<0.05)$.

The volume of tumors in all the groups decreased gradually by increasing the dosage form (Figure 8). The highest dose of SLOS showed the highest inhibition effect after 16 days of treatment. Evaluation of in vivo melanoma study revealed dose and time dependent antitumor activity $(\% \Delta \mathrm{T} / \Delta \mathrm{C})$ in SLOS, EXOS and negative control groups respectively.
Antitumor analysis showed non-formulated topical EXOS gel has shown $58.95 \pm 5.7 \%$ tumor inhibition after applying equivalent concentration of $1000 \mathrm{mg} / \mathrm{kg}$ of treatment. SLOS of same dosage form revealed $97.07317 \pm 0.6 \%$ tumor inhibition. The current results revealed that high concentration of SLOS could significantly suppress the growth of the melanoma tumor compared with non-formulated gel of O.S extract and negative control.

\section{Histopathological Examination}

Anti-melanoma effect of SLOS compared to EXOS gel was evaluated by histological analysis of stained tumor cross sections. Figure 10 demonstrated the histology of tumors excised from animals treated with empty ethosome as negative control which revealed minimum necrosis compared to other groups with plenty of intra tumor blood E087-E096 | ANGIOTHERAPY | Published online February 4, 2019 

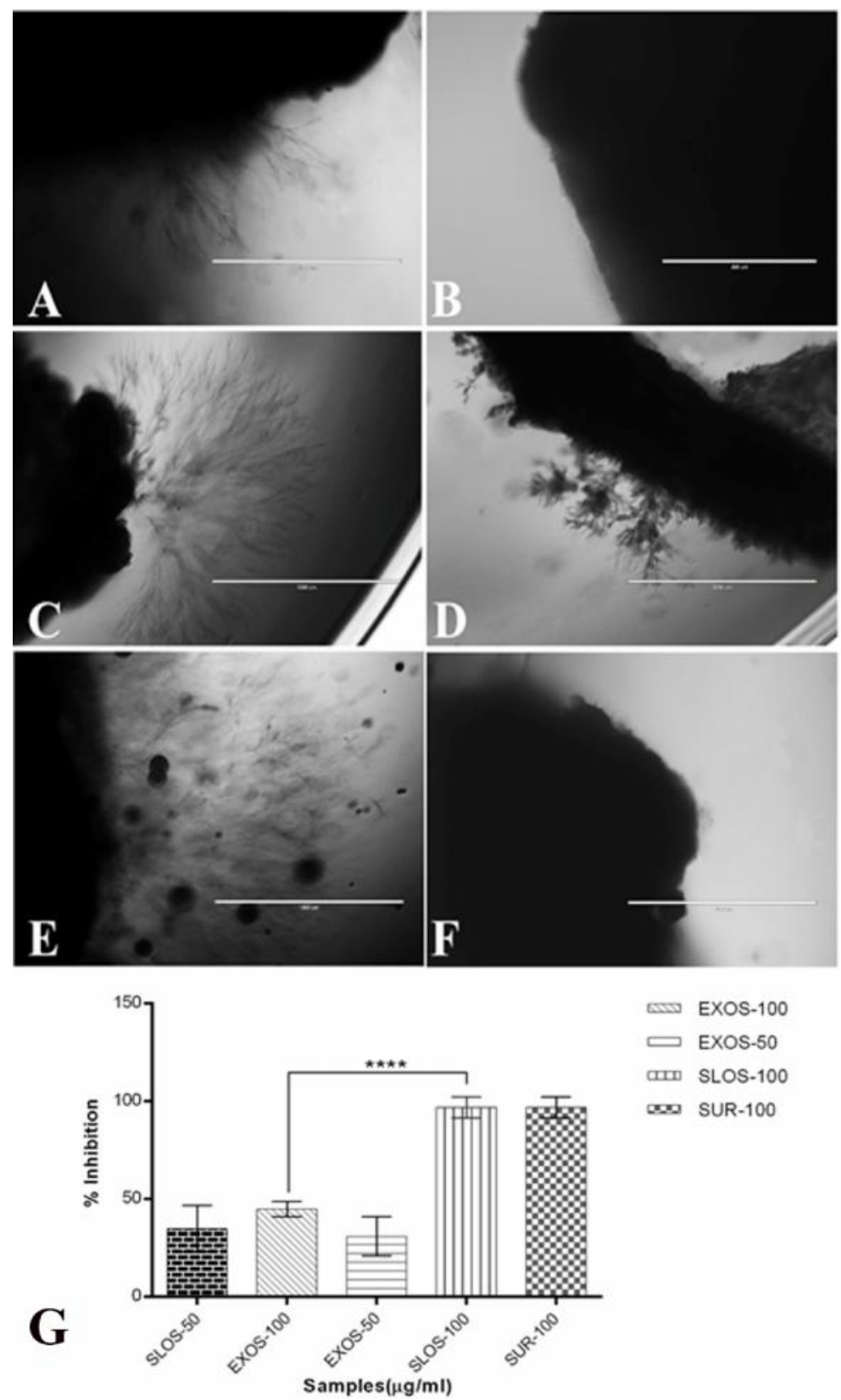

Figure 7 | (A, B) EA.hy926 cell line treated with SLOS at concentration 50 and $100 \mu \mathrm{g} / \mathrm{mL}$.(C,D) treated with EXOS equivalent concetration of 50 and $100 \mu \mathrm{g} / \mathrm{mL}$. (E) negative control. Percentage of EA.hy926 cell line viability after treatment. SLOS and EXOS refer to sophorolipid ethosomal formulation and crude extract of 0.5 , respectively. Statistical analysis was conducted by oneway ANOVA.

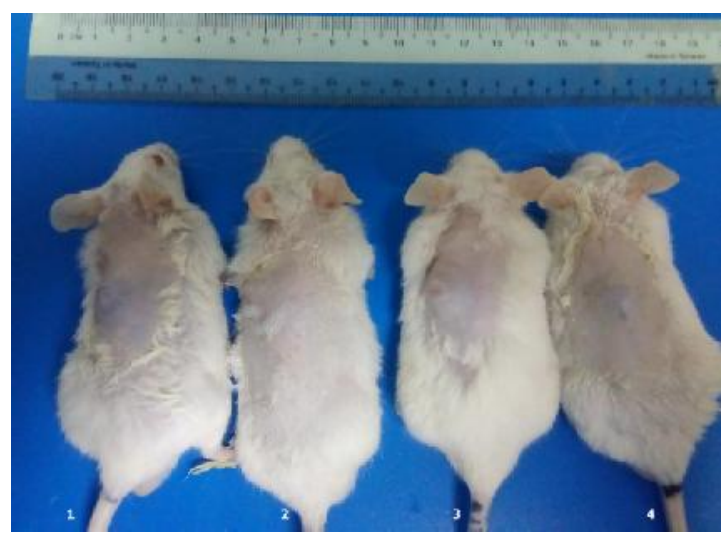

Figure 8 | Anti-melanoma effect of SLOS formulation in B16F10 tumor implanted in mice. $(1,2)$ are negative control at day 0 of treatment. $(3,4)$ are treated group with formulation at day 0 of treatment

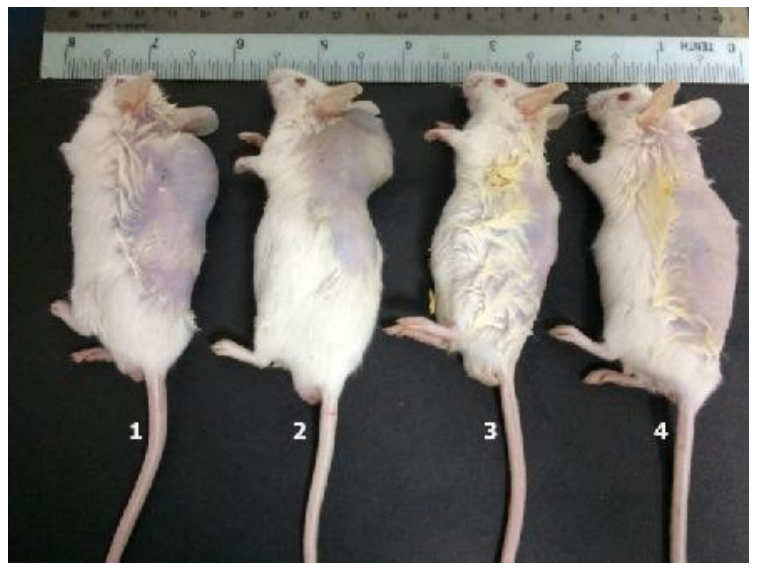

Figure 9 | Anti-melanoma effect of SL-OS formulation in B16F10 tumor implanted in mice. (1) is the animal treated with EXOS, (2) is negative control after 16 days. $(3,4)$ are treated group with SLOS after 16 days treatment. 


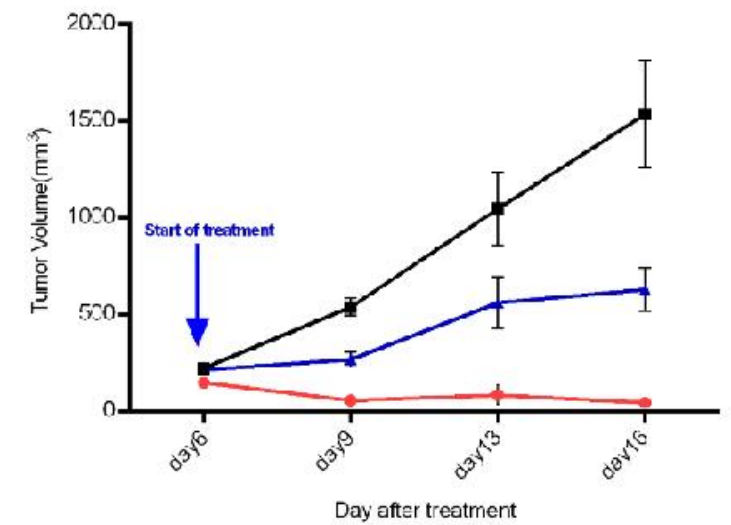

A

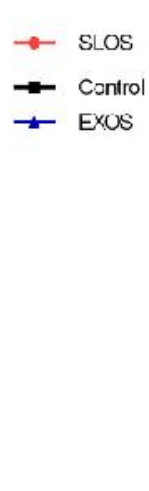

B

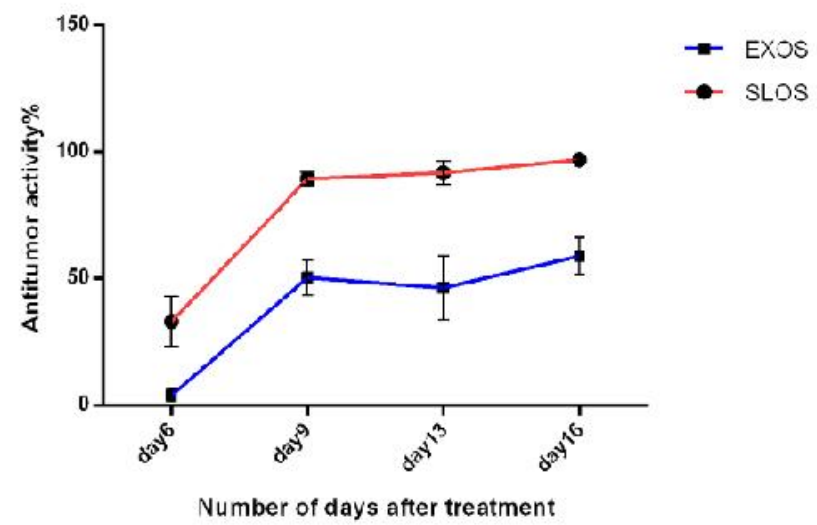

B

Figure 10 | Subcutaneous melanoma tumor in albino mice. (Control) Untreated animals, owing the largest size of tumor, (EXOS) animals after topical treatment with crude extract gel at concentration $1000 \mathrm{mg} / \mathrm{kg}$ body weight. (SLOS) Animals after topical treatment with ethosomal gel at equivalent concentration. (A)Tumor dimensions were analysed every three days and values are demonstrated as mean melanoma tumor size(mm3) \pm S.E.M. (B) shows the antitumor percentage of $1000 \mathrm{mg} / \mathrm{kg}$ body weight of SLOS compared with equivalent concentration of EXOS 16 days treatment in animals. All the studies are demonstrated as mean \pm S.E.M.
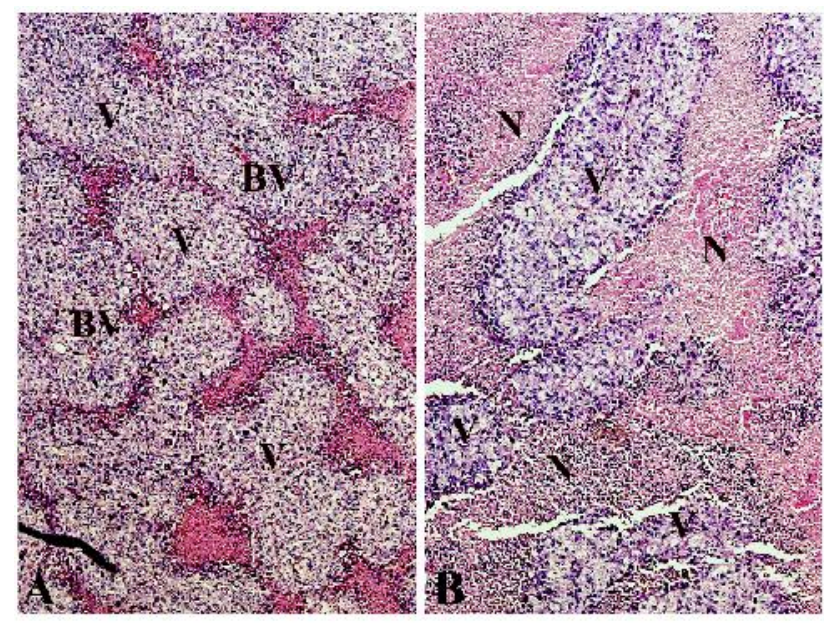

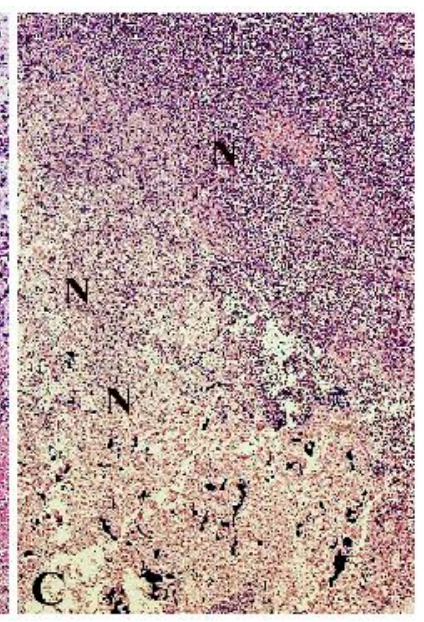

Figure 11 | Cross sections of melanoma tumor tissues stained with hematoxylin/eosin. The tumor cross sections were evaluated for signs of necrosis. (A) The viable melanoma cells and lower extent of necrosis compared to all treated groups, blood vessel and melanin distribution in negative group. (B) The tumor treated with equivalent concentrations at $1000 \mathrm{mg} / \mathrm{kg}$ of EXOS and (C) $1000 \mathrm{mg} / \mathrm{kg}$ of SLOS. V stands for live tumor cells, $\mathbf{N}$ sands for necrosis, BV stands for blood vessels. All the images have been taken at 20x magnification. vessels supply. In the animals treated with SLOS at 1000, 2000 and $3000 \mathrm{mg} / \mathrm{kg}$, the tumors showed increasing trend of necrosis by increasing the dosage of treatment. The cross sections of the tumors in different groups demonstrated disorganized cells, necrosis and reduction of number of blood vessels. Tumor sections in control group demonstrated intact melanoma solid tumor tissues with just small necrotic section and vast area of intra-tumor blood vessels (Error! Reference source not found.). Furthermore, the lung cross section of negative group demonstrated large metastatic tumor cells (Error! Reference source not found.) distribution of melanin in metastasized lung which is more than all treated groups of animal tumors.

\section{Conclusions}

The current study reports the development of a novel sophorolipid ethosomal based gel formulation of O.S extract with high drug-

https://doi.org/10.25163/ angiotherapy.1120591141830219 entrapment capability, effective viscoelasticity, remarkable stability and efficient penetrability. In this study, a gel formulation of ethosome composed of Orthosiphon stamineus extract was optimized with $77.23 \pm 5.9 \%$ entrapment. Flow ramp test study revealed that $1 \%$ gel demonstrated strong potential with optimum viscosity for topical gel formulation. Cytotoxic test on normal cell line confirmed the safety of SLOS in vitro. Physicochemical analysis confirmed the presence of the active compounds in SLOS. Vesicles were developed with smooth surface, which could help in holding up of the drug, prevented the drug-leakage, and thus enhanced permeation efficacy compared with conventional hydroethanolic gel composed of the EXOS. In vivo melanoma tumor model in mice also confirmed the enhanced effect of SLOS compared with EXOS. Altogether, it can be concluded that as SLOS gel is a novel topical formulation that can be a promising treatment methodology for skin cancer.

E087-E096 | ANGIOTHERAPY | Published online February 4, 2019 


\section{Acknowledgement}

The authors would like to express their gratitude to Universiti Sains Malaysia, EMAN Biodiscoveries Sdn. Bhd. and NatureCeuticals Sdn. Bhd. companies for providing research facilities.

\section{References}

Bragagni, M., Scozzafava, A., Mastrolorenzo, A., Supuran, C. T., \& Mura, P. (2015). Development and ex vivo evaluation of 5-aminolevulinic acid-loaded niosomal formulations for topical photodynamic therapy. International Journal of Pharmaceutics, 494(1), 258-263.

Hossain, M. A., \& Rahman, S. M. (2015). Isolation and characterisation of flavonoids from the leaves of medicinal plant Orthosiphon stamineus. Arabian Journal of Chemistry, 8(2), 218-221.

lizhar, S. A., Syed, I. A., Satar, R., \& Ansari, S. A. (2016). In vitro assessment of pharmaceutical potential of ethosomes entrapped with terbinafine hydrochloride. Journal of advanced research, 73), 453-461.

Jain, S., Patel, N., Madan, P., \& Lin, S. (2016). Formulation and rheological evaluation of ethosome-loaded carbopol hydrogel for transdermal application. Drug development and industrial pharmacy, 42(8), 1315-1324.

Li, K., Gao, S., Tian, B., Shi, Y., Lv, Q., \& Han, J. (2018). Formulation Optimization and Invitro and In-vivo Evaluation of Lornoxicam Ethosomal Gels with Penetration Enhancers. Current drug delivery, 15(3), 424-435.

Malla, B., Malla, K., Sah, A. K., Koirala, A., Karki, S., Prajuli, D. R., \& Thapa, B. (2016) Preparation and Characterization of Liposomes and Ethosomes Bearing Indomethacin for Topical drug delivery.

Mbah, C. C., Builders, P. F., Agubata, C. O., \& Attama, A. A. (2017). Development of ethosomal vesicular carrier for topical application of griseofulvin: effect of ethanol concentration. Journal of Pharmaceutical Investigation, 1-10.

Ng, K.-W., Salhimi, S. M., Majid, A. M. S. A., \& Chan, K.-L. (2010). Anti-angiogenic and cytotoxicity studies of some medicinal plants. Planta medica, 76(09), 935-940.

Ramadon, D., Goldie, A. W., \& Anwar, E. (2017). Novel transdermal ethosomal gel containing green tea (Camellia sinensis L. Kuntze) leaves extract: formulation and in vitro penetration study. Journal of Young Pharmacists, 9(3), 336.

Rezaei-Sadabady, R., Eidi, A., Zarghami, N., \& Barzegar, A. (2016). Intracellular ROS protection efficiency and free radical-scavenging activity of quercetin and quercetin-encapsulated liposomes. Artificial cells, nanomedicine, and biotechnology, 44(1), 128-134.

Shafaei, A., Ab Halim, N. H., Zakaria, N., \& Ismail, Z. (2017). Analysis of free amino acids in different extracts of Orthosiphon stamineus leaves by high-performance liquid chromatography combined with solid-phase extraction. Pharmacognosy magazine, 13(Suppl 3), S385.

Shafaei, A., Sultan Khan, M. S., Aisha, A. F., Abdul Majid, A. M. S., Hamdan, M. R., Mordi, M. N., \& Ismail, Z. (2016). Flavonoids-rich Orthosiphon stamineus extract as new candidate for angiotensin I-converting enzyme inhibition: A molecular docking study. Molecules, 21(11), 1500.

Vishkaei, M. N., Sultan, S. B. S. H., Basheer, M. K. A., \& Majid, A. M. (2017). SKIN BARRIER CHALLENGES IN DERMAL AND TRANSDERMAL DRUG DELIVERY SYSTEMS.

Vishkaei, M. N., Sultan, S. B. S. H., Khadeer, M. B., \& Ahamed, A. M. (2017). World Journal of Pharmaceutical Sciences. Regulation, 15, 7.
Submit your next manuscript to Journal of Angiotherapy published by EMAN Research

- Convenient online submission

- Thorough peer review

- No space constraints or color figure charges

- Immediate publication on acceptance

- Inclusion in Australian National Libraray and Google Scholar

- Both Open (80-100\% subsidized APC by ER) \& non-open access option

Submit your manuscript at

https://angiotherapy.emanresearch.org 